\title{
Quantifying Size Errors of Particle Distribution Analysis Using Flatbed Scanner Images.
}

\author{
Jacob L. Mey ${ }^{*}$, Sonali Shukla ${ }^{* * *}$, Edmond A. Mathez* . \\ *American Museum of Natural History, New York, NY 10024, USA \\ ${ }^{* *}$ New York University (NYU), New York, NY 10011, USA
}

Small particle $(100-500 \mu \mathrm{m})$ counting and sizing can be a lengthy process involving numerous high resolution scanning electron microscope (SEM) or backscattered electron (BSE) images and subsequent digital image analysis. A decent quality flatbed scanner offers an easy and inexpensive method for counting a large number of small particles in minutes. One of the advantages is the much larger surface area for sample distribution compared to that of a small slide or stub. As state of the art flatbed scanners become cheaper and computers perform better, even very large ( $\sim 0.5 \mathrm{~GB})$, high-resolution images of particles are easily acquired and analyzed. Sizing artifacts, however, caused by the threshold function during conversion to binary bitmaps are introduced if particles are scanned at insufficient resolution. The purpose here is to quantify these errors, which are typically minor when scanning particles $>500 \mu \mathrm{m}$ at 3600 dots per inch (dpi), but are apparent for particles in the size range of $100-400 \mu \mathrm{m}$ at the same resolution. At 3600 dpi, a round $100 \mu \mathrm{m}$ particle is represented by approx. 154 square pixels ( $\mathrm{r} \sim 7$ pixels) (Fig. 1). The pixel area representing the real sphere is sensitive to sizing errors by eroding (black on Fig. 1) or dilating (grey on Fig. 1) one pixel layer of the circumference as a result of applying too high or too low threshold values when converting to a binary image. In this rather extreme case (Fig. 1), the result is an area size decrease of $\sim 26 \%$ (black) and a size increase of $\sim 23 \%$ (gray) respectively.

High resolution images, each representing 4000 small glass beads with a known calibrated size distribution (NIST SRM 1018b Glass Beads, 220-750 $\mu \mathrm{m}$; NIST SRM 1017b Glass Beads, 100-400 $\mu \mathrm{m}$ ) where scanned with an Epson Expression XL 1680 flatbed scanner at various resolutions (1800, 2800, 3600 and $4200 \mathrm{dpi})$ using various threshold values (160-220 in steps of 5) for binary image acquisition (Fig. 2). The images where analyzed using ImageJ (www.nih.gov), and the particle data were converted to glass masses of volumes of prolate spheroids allowing direct comparison with the NIST calibration curve of the glass beads (Fig. 3). The results quantify the extent of the sizing errors of the particles as a function of the threshold values and image resolution. As expected, the increasing threshold values increasingly affect the data set with decreasing sizes (Fig. 2), but have little effect on larger grains. From the complete data set, a formula has been derived estimating the size errors as a function of resolution and threshold values. This function can be used as a guide for choosing the best parameters when scanning particles of various size ranges using this technique. 


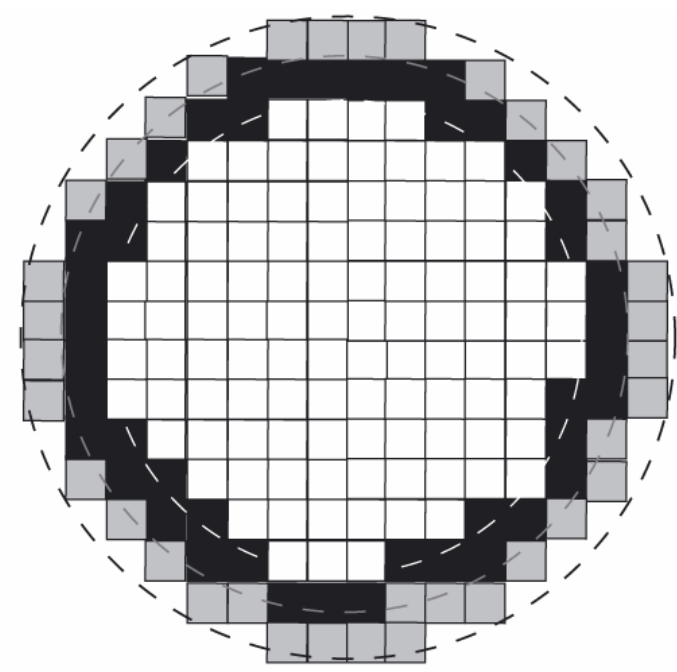

Figure 1: The effect of dilation (gray) and erosion (black) of too extreme threshold on a $100 \mu \mathrm{m}$ grain image, (scanned at $3600 \mathrm{dpi}$ ) during conversion to a binary image.

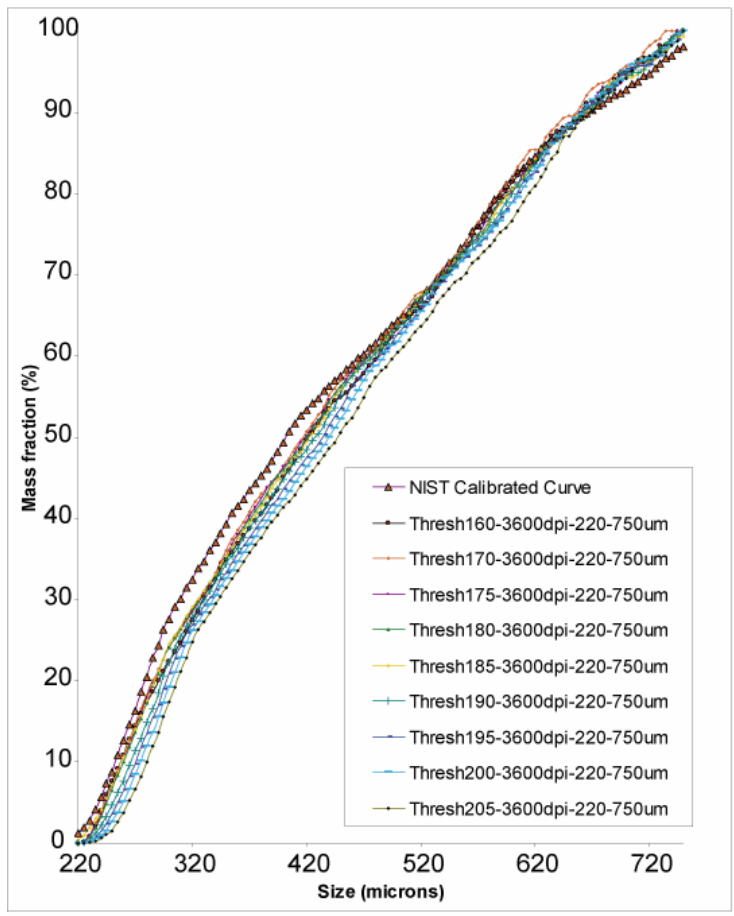

Figure 3: Size distribution curves from images with various threshold values compared to known distribution of NIST SRM $1018 \mathrm{~b}$ Glass Beads ( $n \sim 4000$ glass beads for each curve)
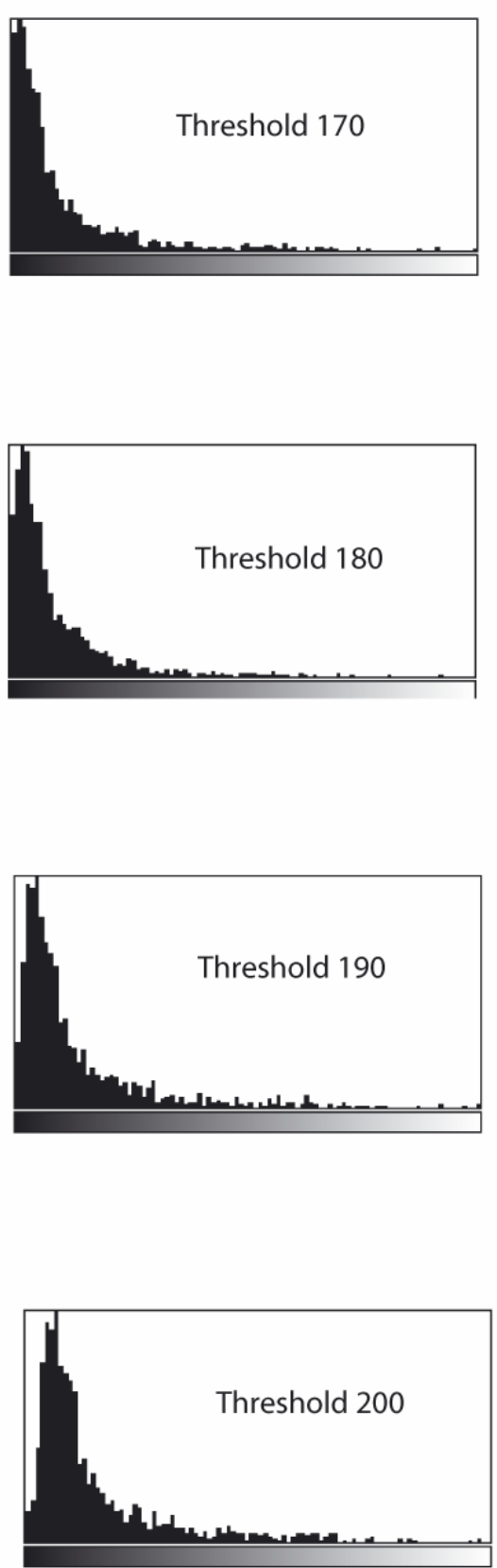

Figure 2: The effects of increasing value of binary threshold on the distribution of particle sizes ( $\mathrm{n} \sim 4000$ glass beads, sizes range from 200 to $800 \mu \mathrm{m})$. 